\section{European Society for Dermatology and Psychiatry (ESDaP)}

ESDaP

Françoise Poot M.D. (Secretary)

U.L.B.

Hôpital Erasme, Dermatology Department

Route de Lennik 808

B-1070 Brussels

Tel. +32025553007

Sister Society Meeting of the ESDaP:

EADV 13th Congress, Florence, November 17, 2004,

\section{Fortezza da Basso}

$14.00 \mathrm{~h}$

Introduction

Emiliano Panconesi, ESDaP-President, Florence, Italy

$14.15 \mathrm{~h}$

Quality of Life in Patients with Post Herpetic Neuralgia

Lucia Tomas Aragones, Zaragoza; Servando E. Marron,

Moya, Spain

$14.45 \mathrm{~h}$

Coping Strategies and Quality of Life in Psoriasis Patients

Anna Zalewska, Lodz, Poland

$15.15 \mathrm{~h}$

Body Dysmorphic Disorder

Uwe Gieler, Giessen, Germany

$15.45 \mathrm{~h}$

What's New in Psychodermatology?

Françoise Poot, Brussels, Belgium

$16.00 \mathrm{~h}$

How Do I Organize My Psychodermatology Consultation?

Uwe Gieler, Giessen, Germany; Françoise Poot, Brussels,

Belgium

$16.30 \mathrm{~h}$

Case Presentations and Discussion with the Speakers and the Audience

Open to the participants

$17.00 \mathrm{~h}$

End of the Session

\section{Société Francophone de Dermatologie Psychosomatique (SFDPS)}

SFDPS

Dr. Sylvie Consoli

7, rue du Mouton Duvernet

F-75014 Paris

Tél. 01404446 02, Fax 0146613412

\section{Cours 2004-2005: F.M.C. de la SFDPS}

Lieu:

Hôpital Européen Georges Pompidou

20, rue Leblanc

Paris 15ème

Dates:

16-17 octobre 2004: l'hystérie (Auditorium 1er étage, 15.45 h) 12-13 mars 2005: les dysmorphophobies (Salle du Conseil 8 è étage, 15.45 h)

12 mars 2005: assemblée générale de la SFDPS (19.30 h)

Renseignements:

Pascale Van Vaeck

35 , rue des Baconnets

F-92160 Anthony

\title{
KARGER
}

(C) 2004 S. Karger GmbH, Freiburg 


\section{Institut de Formation et de Thérapie pour Soignants (IFTS)}

\section{IFTS}

Rue de la Flanière 44

B-6043 Charleroi

Tel./Fax +32 71354344

www.ifts.be

\section{Formation en psychosomatique}

Diplôme de formation à la prise en charge des maladies psychosomatiques

Dates: 02/10/2004; 04/12/2004; 19/02/2005; 05/03/2005; 23/04/2005; 14/05/2005; 08/10/2005; 17/12/2005; 04/02/2006; $18 / 03 / 2006 ; 15 / 04 / 2006 ; 20 / 05 / 2006$
Supervision en psychosomatique

Dates: 11/09/2004; 15/01/2005; 18/06/2005; 17/09/2005;

14/01/2006; 10/06/2006

Formation au génogramme (obligatoire pour le diplôme)

Dates: En 2004 le 27/11/2004; en 2005 le 19/11/2005

\section{Summer Training in Psychodermatology}

Aims: To acquire knowledge and tools to take care of psychosomatic dermatologic patients together with better communication skills.

Conditions: To be a dermatologist or a psychotherapist.

Venue: IFTS, rue de la Flanière 44, B-6043 Charleroi, Belgium (very close to Brussels South airport).

Dates: July 7-8, 2005

Information:www.ifts.be; f.poot@yucom.be 